\title{
Hypotrichosis with juvenile macular degeneration
}

INSERM

\section{Source}

INSERM. (1999). Orphanet: an online rare disease and orphan drug data base.

Hypotrichosis with juvenile macular degeneration. ORPHA:1573

Hypotrichosis with juvenile macular degeneration (HJMD) is a very rare syndrome characterized by sparse and short hair from birth followed by progressive macular degeneration leading to blindness. 